\title{
NEAR-INFRARED OBSERVATIONS OF A REDSHIFT 4.92 GALAXY: EVIDENCE FOR SIGNIFICANT DUST ABSORPTION ${ }^{1}$
}

\author{
B. T. Soifer, ${ }^{2}$ G. Neugebauer, ${ }^{2}$ M. Franx, ${ }^{3}$ K. Matthews, ${ }^{2}$ and G. D. Illingworth ${ }^{4}$ \\ Received 1997 December 5; accepted 1998 May 15; published 1998 June 26
}

\begin{abstract}
Near-infrared imaging and spectroscopy have been obtained of the gravitationally lensed galaxy at $z=4.92$ discovered in Hubble Space Telescope (HST) images by Franx et al. Images at 1.2, 1.6, and $2.2 \mu \mathrm{m}$ show the same arc morphology as the HST images. The spectrum with resolution $\lambda / \Delta \lambda \sim 70$ shows no emission lines with equivalent width stronger than $100 \AA$ in the rest-frame wavelength range $0.34-0.40 \mu \mathrm{m}$. In particular, [O II] $\lambda 3727$ and [Ne III] $\lambda 3869$ are not seen. The energy distribution is quite blue, as expected for a young stellar population with the observed $\operatorname{Ly} \alpha$ flux. The spectral energy distribution can be fitted satisfactorily for such a young stellar population when absorption by dust is included. The models imply a reddening $0.1 \mathrm{mag}<$ $E(B-V)<0.4 \mathrm{mag}$. The stellar mass of the lensed galaxy lies in the range of $2-16 \times 10^{9} M_{\odot}$. This is significantly higher than estimates based on the HST data alone. Our data imply that absorption by dust is important to redshifts of $\sim 5$.
\end{abstract}

Subject heading: galaxies: formation — galaxies: starburst

\section{INTRODUCTION}

The study of galaxies at high redshifts has been revolutionized with the introduction of the Hubble Space Telescope (HST) and the new generation of large ground-based telescopes. Detecting and studying such systems at high redshift is essential to understanding how normal galaxies form and evolve. Franx et al. (1997) have recently reported the serendipitous discovery of a field galaxy at a redshift $z=4.92$ that has been gravitationally lensed into several arc components, including a highly magnified fold arc, by an intervening cluster of galaxies (CL $1358+62)$ at $z=0.33$. Franx et al. present $H S T$ imaging and Keck spectroscopy of this highly magnified lensed system. Because of the redshift, the $H S T$ images at $R(\mathrm{~F} 606 \mathrm{~W})$ and $I$ (F814W) correspond to wavelengths of 1023 and $1375 \AA$ in the rest frame of the background objects and thereby sample only the far-UV portion of the light emitted from these galaxies. In order to study these objects at wavelengths where nearby galaxies have been studied, we observed the brightest (fold) arc of the lensed galaxy at near-infrared wavelengths using the W. M. Keck Telescope. In the discussion we adopt the same cosmological parameters as in Franx et al., i.e., $H_{0}=50 \mathrm{~km}$ $\mathrm{s}^{-1} \mathrm{Mpc}^{-1}$ and $q_{0}=0.5$.

\section{OBSERVATIONS AND DATA REDUCTION}

The observations reported here were made in 1997 April in photometric conditions using the near-infrared camera (NIRC) at the $f / 25$ forward Cassegrain focus of the W. M. Keck Telescope. The instrument is described in detail by Matthews \& Soifer (1994). It has a $256 \times 256 \mathrm{InSb}$ array with $0^{\prime \prime} .15 \times 0$ 0"15 pixels for a $38^{\prime \prime} \times 38^{\prime \prime}$ field of view.

Sets of images centered on the brightest portion of the arc (locations B to C, described by Franx et al. 1997) were obtained at $1.25 \mu \mathrm{m}(J), 1.65 \mu \mathrm{m}(H)$, and $2.2 \mu \mathrm{m}(K)$. The broadband $J, H$, and $K$ images were made with total exposures of 1620

\footnotetext{
${ }^{1}$ Based on observations obtained at the W. M. Keck Observatory.

${ }^{2}$ Palomar Observatory, 320-47, Caltech, Pasadena, CA 91125.

${ }^{3}$ Kapteyn Institute, P.O. Box 800, NL-9700 AV, Groningen, The Netherlands.

${ }^{4}$ University of California Observatories/Lick Observatory, Board of Studies in Astronomy and Astrophysics, University of California, Santa Cruz, CA 95064.
}

$\mathrm{s}$ per filter. In addition, a set of images was taken in a $1.2 \%$ bandpass filter centered at a wavelength of $2.297 \mu \mathrm{m}$ (rest wavelength $3881 \AA$ ), which contained the redshifted [Ne III] $\lambda 3869$ line). The total exposure time at this wavelength was $2700 \mathrm{~s}$. The imaging data were obtained in sets of $60 \mathrm{~s} \mathrm{ex-}$ posures, where the center of the field was moved in a $3 \times 3$ grid pattern $10^{\prime \prime}$ on a side. The centers of successive grids were moved by $1 "-2$ " between each set. Bright cluster galaxies in the frames were used to accurately register the successive frames for co-addition.

Sky and normalized flat-field frames were created from the data at each wavelength separately, taking the clipped mean of nine frames centered in time on the frame of interest. The seeing, as determined from several stellar images in the field, was approximately 0".5-0".6 FWHM. The data were calibrated using calibration stars of Persson (1998).

In addition to the images, low-resolution grism spectra were obtained of selected sources within the arc. These spectra were obtained over the wavelength range 1.5-2.4 $\mu \mathrm{m}(0.25-0.40 \mu \mathrm{m}$ in the rest frame). The slit was $0^{\prime \prime} .7$ wide, yielding a spectral resolution of $\lambda / \Delta \lambda \sim 70$. Slit position angles of $80^{\circ}$ and $145^{\circ}$ were used. The brightest portion of the fold arc $\mathrm{C}$, the knot and the northwest end (Franx et al. 1997) was contained in the slit for the P.A. $=80^{\circ}$ spectrum, while the slit was aligned with the arc for the P.A. $=145^{\circ}$ spectrum. For each position angle the integration time was $3000 \mathrm{~s}$. The knot at the NW end of arc $\mathrm{C}$ was chosen because it was the brightest of the lensed knots, although the data of Franx et al. show that the strength of $\operatorname{Ly} \alpha$ is clearly lower at that position (the equivalent width of $\operatorname{Ly} \alpha$ at this location drops to $1 / 3$ of its value elsewhere in the arc).

\section{RESULTS}

The $J$ image of the field of CL $1358+62$ is shown in Figure 1 (top). Locations B and $\mathrm{C}$ in the nomenclature of Franx et al. (1997) are labeled. The arc is seen in the $J, H$, and $K$ images over the entire spatial range in which it is visible in the HST images. The arc was also detected in the narrowband image at a level consistent with the $K$-band continuum image and shows that the $[\mathrm{Ne}$ III] line does not contribute significantly to the flux (equivalent width less than $50 \AA$ ) in the arc. 

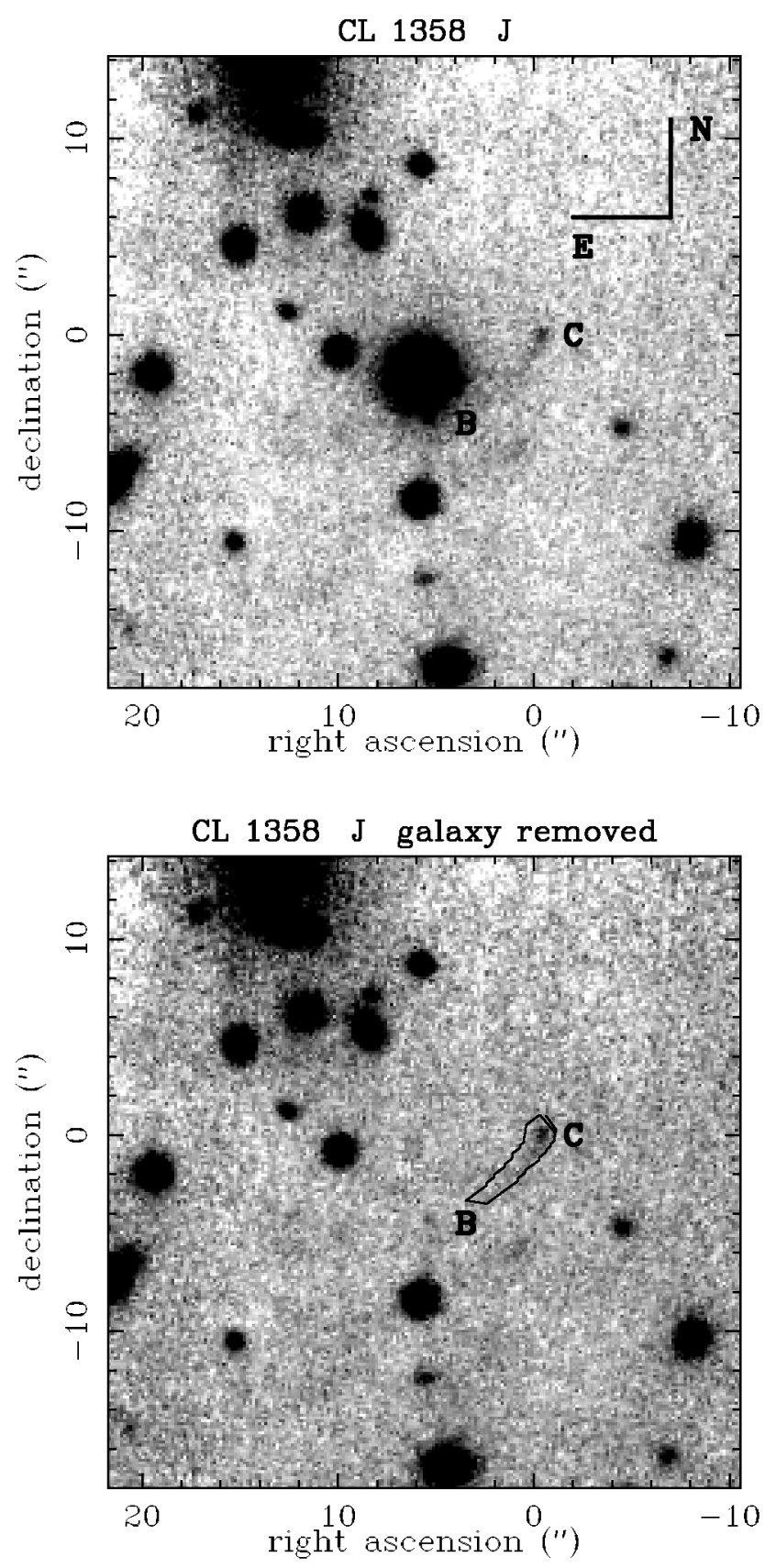

FIG. 1.-Image of lens arc associated with the cluster CL $1358+62$ in the $J$ band obtained with the W. M. Keck Telescope. The top panel is the image of the field with the orientation indicated in the image, the bottom panel is the same image with the lensing galaxy removed. Locations B and C from Franx et al. are indicated in the top image, while the outline of the photometric aperture $\mathrm{C}$ is shown in the bottom image.

In order to directly compare the Keck images with the HST image, we have convolved the HST I image with the measured seeing of the Keck data. In Table 1 we report photometry at $I, J, H$, and $K$ in three beams; position 1 is a circular beam including the brightest peak in the arc at position $\mathrm{C}$, position 2 includes the full arc from $B$ to $C$ with the beam shaped to mimic the arc but excluding the brightest knot, and position 3 is the sum of the first two beams. Figure 1 (bottom) shows the shape of the beam of position 3 .

To derive photometry that is less sensitive to the presence of the bright elliptical galaxy north-northeast of the fold arc, we first determined the surface brightness profile of this galaxy in each of the images using an ellipse-fitting program. We subtracted the resulting model distribution from the images. This produced a flat background at the location of the arc that reduced the uncertainties in the photometry. The $J$-band image formed in this way is shown in Figure 1 (bottom). To minimize the uncertainties in the photometry, we made the sky beam as large as possible while avoiding the field objects near the arc. The photometry obtained with and without the elliptical galaxy removed from the image was indistinguishable at all wavelengths. Because we felt the results were more robust, the photometry with the elliptical galaxy removed is reported in Table 1.

The spectrum at P.A. $=80^{\circ}$, taken with the bright knot of the fold arc $\mathrm{C}$ centered in the slit, shows that the continuum was detected at low signal-to-noise ratio. No convincing detection of the arc was made in the spectrum at P.A. $=145^{\circ}$. No lines were detected in either spectrum over the wavelength range $0.25-0.40 \mu \mathrm{m}$ in the rest frame of the object. This corresponds to an upper limit on the equivalent width of $100 \AA$ for any emission line over the wavelength range $0.34-0.40 \mu \mathrm{m}$ and a limit of $200 \AA$ in the equivalent width of any emission line over the wavelength range $0.25-0.34 \mu \mathrm{m}$ (the higher limit at shorter wavelengths is a result of the strong $\mathrm{OH}$ airglow emission in the $H$ band).

Since there is at best only marginal evidence for any color difference between positions 1 and 2, we adopt the highest signal-to-noise measurements of the sum of these apertures (position 3) to determine the intrinsic energy distribution of the source galaxy. This was determined by measuring the colors $I-[\lambda]$ at position 3 and subtracting these colors from the intrinsic $I_{A B, 814}=24.0 \mathrm{mag}$ of the lensed galaxy G1 determined by Franx et al. (1997). This leads to intrinsic (unmagnified) magnitudes for G1 of 22.4, 22.3, and $21.5 \mathrm{mag}$ at $J, H$, and $K$ respectively. We do not believe that the quality of the data warrant interpreting the results in terms of color variations across the lensed object. In the discussion that follows the derived quantities refer to the intrinsic (unlensed) galaxy.

\section{STELLAR POPULATION MODELS}

The near-infrared wavelengths correspond to rest-frame UV and blue in the lensed galaxy, with the $J, H$, and $K$ images being at $2150 \AA, 2790 \AA$, and $3720 \AA$, respectively. The broadband colors potentially suffer from contamination by strong emission lines. In the $K$ band the strongest potential contamination is a result of [O II] $\lambda 3727$ emission. The limit of 100 $\AA$ on the rest-frame equivalent width of the [O II] line in the spectrum of the bright knot of $\mathrm{C}$ limits the contamination of the $K$ continuum to less than $20 \%$ of the total observed flux in the band. This limit is consistent with that seen in young star-forming galaxies at $z \sim 1$ by Cowie, $\mathrm{Hu}, \&$ Songaila (1995), who find an average rest-frame equivalent width in [O II] of $60 \AA$ A. The limit on [O II] equivalent width limits the increase in the spectral index because of line contamination in $K$ to $\Delta \beta<0.3$, where the spectral index is defined as $F_{\lambda} \propto$ $\lambda^{\beta}$. In the $H$ band, the strongest potential contaminant is Mg II $\lambda 2800$, while in the $J$ band it is C III] $\lambda 1909$. Our limit on the $\mathrm{Mg}$ II line is not particularly significant, while we do not have spectra covering the $\mathrm{C}$ III] wavelength. The $\mathrm{C}$ III] and $\mathrm{Mg}$ II lines are not seen in the nuclear spectra of nearby starbursts such as NGC 4214 (Leitherer et al. 1996) or blue compact galaxies such as Haro 2 (Thuan 1990), while our limit on $[\mathrm{O}$ II] $\lambda 3727$ is consistent with the strongest lines seen in nearby 
TABLE 1

Рhotometry of Locations in Lensed ARC In CL 1358+62

\begin{tabular}{llccccc}
\hline \hline Position & \multicolumn{1}{c}{ Beam } & $\begin{array}{c}I^{\mathrm{a}} \\
(\mathrm{mag})\end{array}$ & \multicolumn{1}{c}{$\begin{array}{c}J \\
(\mathrm{mag})\end{array}$} & $\begin{array}{c}H \\
(\mathrm{mag})\end{array}$ & $\begin{array}{c}K \\
(\mathrm{mag})\end{array}$ \\
\hline $1 \ldots \ldots$. & Circ $^{\mathrm{b}}$ & $22.88 \pm 0.04$ & $21.7 \pm 0.2$ & 21.6 & \pm 0.3 & $21.3 \pm 0.3$ \\
$2 \ldots \ldots$. & Arc $^{\mathrm{c}}$ & $22.79 \pm 0.04$ & $21.84 \pm 0.20$ & $21.40 \pm 0.30$ & $20.54 \pm 0.18$ \\
$3 \ldots \ldots$. & Irr $^{\mathrm{d}}$ & $22.11 \pm 0.03$ & $20.92 \pm 0.11$ & $20.83 \pm 0.22$ & $19.99 \pm 0.14$ \\
\hline
\end{tabular}

${ }^{\text {a }}$ The magnitude referred to here as $I$ is that from the $H S T$ image in the $\mathrm{F} 814 \mathrm{~W}$ filter from the data of Franx et al. 1997.

${ }^{\mathrm{b}}$ Photometry refers to a 1".8 diameter circular beam, centered on object C (in nomenclature of Franx et al.).

${ }^{c}$ Photometry refers to arc-shaped beam that includes object B and the arc between B and $\mathrm{C}$ (in nomenclature of Franx et al.).

${ }^{\mathrm{d}}$ Photometry refers to an irregularly shaped beam that includes both objects $\mathrm{B}$ and C and the arc between them (the sum of positions 1 and 2).

blue galaxies by Gallagher, Bushouse, \& Hunter (1989). Thus, we conclude that the effect of line emission on the derived colors is at most comparable to the uncertainties in the observations.

The slope of the continuum is well characterized by $F_{\lambda} \propto$ $\lambda^{-1.63}$. Although this is fairly blue, it not as blue as models predict for young, ionizing starburst populations (see, e.g., Leitherer \& Heckman 1995). Most of their models predict a slope of less than -2.2 for ages smaller than $10^{7} \mathrm{yr}$ (as needed for the observed Ly $\alpha$; see below). The redder colors we observe can be produced either by dust, or by the presence of older stars. In both cases the mass of the stellar population would be seriously underestimated if the masses are estimated from the bluest passband. To address this issue, we made fits to the spectral energy distribution using the models by Bruzual \& Charlot $\left(1993,1996^{5}\right)$. We considered models for populations with instantaneous starbursts and with constant star formation rates and have included the effect of reddening.

The results are shown in Figure 2. Instantaneous starburst models with ages between $3 \times 10^{5}$ and $10^{7} \mathrm{yr}$ are shown in Figures $2 a$ and $2 c$. The age of the burst population has to be lower than $10^{7} \mathrm{yr}$ to provide the ionizing flux for the Ly $\alpha$ emission. The IMF was assumed to be a Salpeter law. Figure $2 a$ shows models with no reddening fitted to the data including the $I$ photometry, while Figure $2 c$ shows models fitted to the data where reddening is a free parameter. The models can provide good fits with a dust reddening $E(B-V)$ between 0.26 and 0.37 mag but do not fit the data satisfactorily when reddening is not included. The masses of the stellar population were in the range from 5 to $16 \times 10^{9} M_{\odot}$.

Models with continuous star formation can also provide a good fit to the data once reddening is considered. Figures $2 b$ and $2 d$ show models with ages up to $10^{9} \mathrm{yr}$. We do not consider models with ages of more than $10^{9} \mathrm{yr}$, since this is approximately the Hubble time at $z=4.92$. As with the instantaneous burst models, the models in Figure $2 b$ assume no reddening, while in Figure $2 d$ reddening is a free parameter in the fit, and these models provide significantly better fits than the dust-free models. The required reddening $E(B-V)$ lies between 0.15 and 0.39 mag. The required star formation rates lie between 130 and $4 \times 10^{4} M_{\odot} \mathrm{yr}^{-1}$. The total stellar masses range from 7 to $30 \times 10^{9} M_{\odot}$, for ages from $10^{7}$ to $10^{8} \mathrm{yr}$, respectively. The mass becomes progressively larger with even greater ages. As can be seen in Figure $2 d$, the models with ages $\geq 10^{8}$ yr predict a strong Balmer break, and this significantly reduces

\footnotetext{
${ }^{5}$ The GISSEL Population Synthesis Models are available via anonymous $\mathrm{ftp}$ at gemini.tuc.noao.edu in the directories /pub/charlot/bc95 and /pub charlot/ bc96.
}

the quality of the fit. At an age of $10^{9} \mathrm{yr}$, the total $\chi^{2}$ for the dust-free model is 40 , while that containing dust is 4.6. The observed equivalent width of Ly $\alpha$ between $7 \AA$ and $21 \AA$ does not provide a strong constraint on these continuous star-forming models.

The new data result in much higher mass estimates than the earlier estimates (Franx et al. 1997), which were derived from the rest-frame far-UV flux (observed $H S T I_{814}$ ). The masses are increased because the fluxes in the new redder passbands are significantly higher than predicted by the (dust-free) models used by Franx et al. An additional dependency with these new mass estimates is on the form of the extinction curve used. The Calzetti, Kinney, \& Storchi-Bergmann (1994) extinction curve is rather grey. If we use an SMC extinction curve, we find that the absorption and stellar masses are decreased by a factor of about 2 .

We can also estimate masses by ignoring the extinction and simply using the reddest flux, i.e., the observed $K$-band flux, or rest-frame $U$. We obtain masses from 1.1 to $5 \times 10^{9} M_{\odot}$ for the instantaneous burst models, and masses between 1.6 and $9 \times 10^{9} M_{\odot}$ for the continuous star formation models. These estimates are roughly one-quarter of the estimates derived by using the Calzetti et al. (1994) extinction curve, and about half the estimate derived from the SMC extinction curve. We conclude therefore that the uncertainty in the mass estimate is about a factor of 4 .

A realistic assessment of the likely mass is given by the geometric mean of the no-reddening values and the values based on the Calzetti et al. (1994) extinction curve: masses between $2 \times 10^{9}$ and $9 \times 10^{9} M_{\odot}$ for instantaneous bursts and between $3 \times 10^{9}$ and $16 \times 10^{9} M_{\odot}$ for continuous star formation models.

\section{DISCUSSION}

The discussion of the last section has shown that the lensed galaxy has significant extinction, and we have derived new larger estimates for the stellar mass. The high stellar masses imply that the galaxy has built up a very dense center. The knot, which has an effective radius of $130 \mathrm{pc}$, contains roughly half the mass, i.e., from 1.2 to $8 \times 10^{9} M_{\odot}$ (Franx et al. 1997). Assuming an isothermal profile, we derive a velocity dispersion of the knot between 100 and $260 \mathrm{~km} \mathrm{~s}^{-1}$. Our new results have therefore strengthened the case that this young galaxy at $z=$ 4.92 has already managed to build up a very dense core.

The resulting dynamical timescale for the knot is a few times $10^{6} \mathrm{yr}$, comparable to the age derived from a starburst model. The dynamical timescale for the galaxy as a whole is at least 10 times higher. This exceeds the lifetime of ionizing $\mathrm{O}$ stars. 

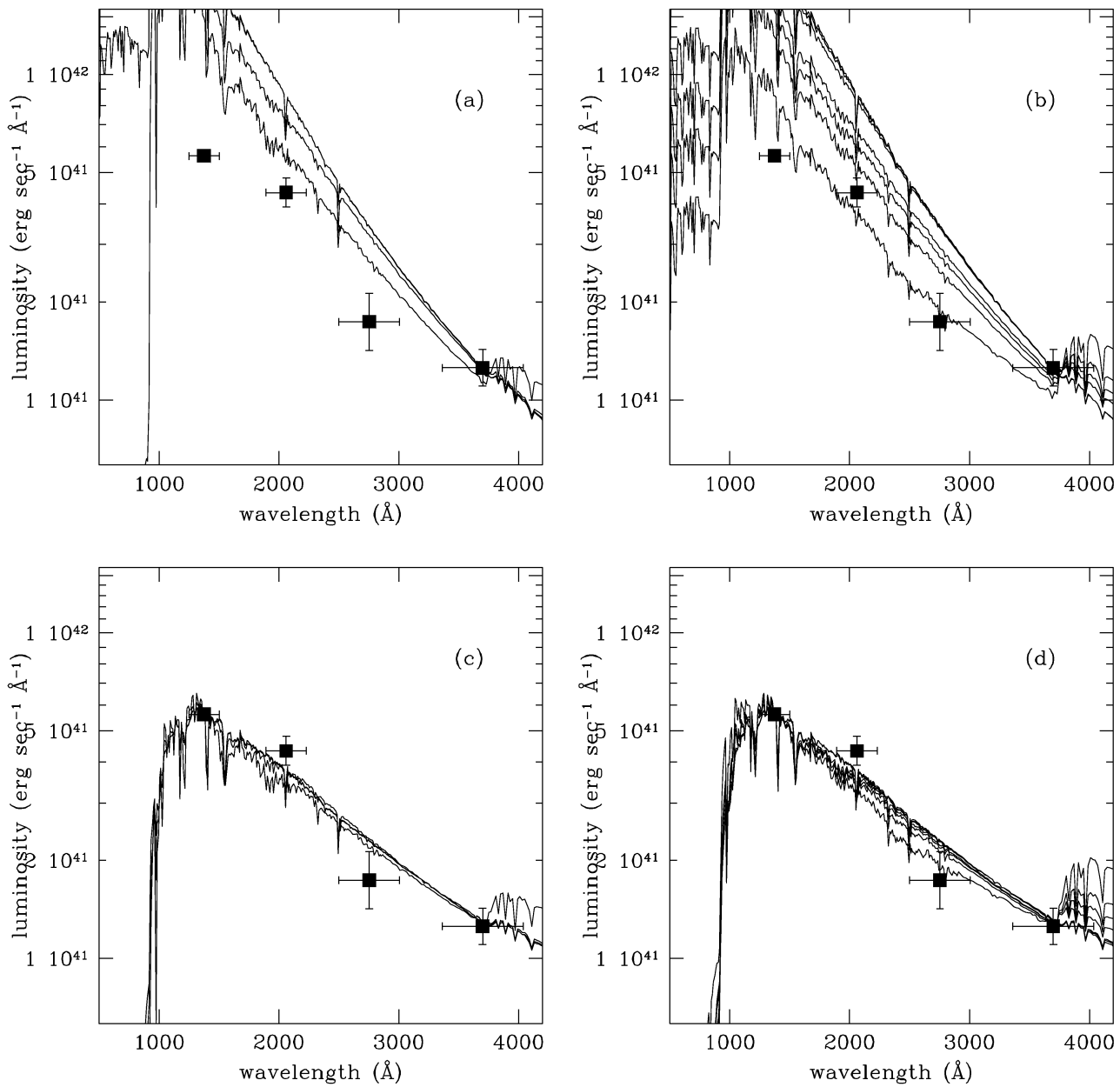

FIG. 2.-Energy distribution for the lensed system in CL $1358+62$, plotted as luminosity density vs. rest wavelength compared with instantaneous and continuous star formation models from Burzual \& Charlot $(1993,1996)$. The left panels, represent instantaneous models of ages 10, 30, and 100 $\times 10^{5} \mathrm{yr}$, the top panel being unreddened, the bottom panel having a reddening $E(B-V)$ between 0.23 and 0.33 mag. The right panels represent continuous star formation models with ages of $3,10,30,100,300,1000$, and $10,000 \times 10^{5}$ yr. The top panel is unreddened models, the bottom panel having reddening between 0.15 and 0.39 mag to best fit the data, with the older models requiring less reddening. For both sets of models, ages below the youngest listed age are indistinguishable.

Combined with the fact that the emission and absorption line properties observed by Franx et al. (1997) indicate the presence of a strong wind that locally depletes the interstellar medium in this galaxy, it is likely that we are seeing young, short-lived condensations of star formation. Over time, these knots would brighten progressively across the galaxy, as suggested earlier by Lowenthal et al. (1997).

The infrared photometry has demonstrated that dust is very likely present in the galaxy. This conclusion clearly depends on the accuracy of the stellar evolutionary models. The reddening is estimated to be between $0.1 \mathrm{mag}<E(B-V)<0.4$ mag, depending on the population model and extinction curve. These values are comparable to those found in nearby, lowmetallicity starbursts (see, e.g., Meurer et al. 1997). This implies that the galaxy has been able to produce a significant amount of metals, but the observations to date are inadequate to derive metallicities.

We note that similar results were derived by Ellingson, Yee, \& Bechtold (1996) for MS 1512-cB58 and for galaxies in the Hubble Deep Field by Sawicki \& Yee (1998). The optical and infrared fluxes of MS 1512-cB58 at $z=2.72$ imply a young age $\left(10^{7} \mathrm{yr}\right)$ and a significant extinction $[E(B-V) \sim 0.3 \mathrm{mag}]$. The constraints on the age of the young population were stronger because the observations extended beyond the Balmer break. In the case of the Hubble Deep Field, Sawicki \& Yee have found that spectral energy distributions with reddening of $E(B-V) \sim 0.3 \mathrm{mag}$ provides better fits to the observations of $z>2$ Lyman Break galaxies than do dust-free models. The best studied high-redshift galaxies have significant amounts of dust, while evidence for dust exists for other high-redshift galaxies based on sparser color information (see, e.g., Meurer et al. 1997; Pettini et al. 1997). If the lensed galaxies are typical, then a reddening between $E(B-V) \approx 0.2-0.3$ mag should be expected for most high-redshift galaxies.

We thank W. Harrison for assistance with the observations, and S. E. Persson for providing photometric standards in advance of publication. The W. M. Keck Observatory is operated as a scientific partnership between the California Institute of Technology, the University of California and the National Aeronautics and Space Administration. It was made possible by the generous financial support of the W. M. Keck Foundation. Infrared astronomy at Caltech is supported by grants from the NSF and NASA. Support from STScI grant GO05989.01-94A is also gratefully acknowledged. This research has made use of the NASA/IPAC Extragalactic Database, which is operated by the Jet Propulsion Laboratory, Caltech, under contract with NASA. 


\section{REFERENCES}

Bruzual, G., \& Charlot, S. 1993, ApJ, 405, 538 1996, GISSEL Population Synthesis Models

Calzetti, D., Kinney, A. L., \& Storchi-Bergmann, T. 1994, ApJ, 429, 582

Cowie, L. L., Hu, E. M., \& Songaila, A. 1995, Nature, 377, 603

Ellingson, E., Yee, H. K. C., \& Bechtold, J. 1996, ApJ, 466, L71

Franx, M., Illingworth, G. D., Kelson, D. D., van Dokkum, P. G., \& Tran, K.V. 1997, ApJ, 486, L75

Gallagher, J. S., Bushouse, H., \& Hunter, D. A. 1989, AJ, 97, 700

Leitherer, C., \& Heckman, T. M. 1995, ApJS, 95, 9

Leitherer, C., Vacca, W. D., Conti, P. S., Filippenko, A. V., Robert, C., \& Sargent, W. L. W. 1996, ApJ, 465, 717
Lowenthal, J. D., et al. 1997, ApJ, 481, 673

Matthews, K., \& Soifer, B. T. 1994, Infrared Astronomy with Arrays: the Next Generation, ed. I. McLean (Dordrecht: Kluwer), 239

Meurer, G. R., Heckman, T. M., Lehnert, M. D., Leitherer, C., \& Lowenthal, J. 1997, AJ, 114,54

Persson, S. E. 1998, in preparation

Pettini, M., Steidel, C. S., Adelberger, K. L., Kellogg, M., Dickinson, M., \& Giavalisco, M. 1997, preprint (astro-ph/9708117)

Sawicki, M., \& Yee, H. K. C. 1998, AJ, 115, 1329

Thuan, T. X. 1990, Massive Stars in Starbursts, ed. C. Leitherer, N. Walborne, T. Heckman, \& C. Norman (Cambridge: Cambridge Univ. Press), 183 